\title{
CBX6 overexpression contributes to tumor progression and is predictive of a poor prognosis in hepatocellular carcinoma
}

\author{
Hao Zheng ${ }^{1, *}$, Wei-hua Jiang ${ }^{2, *}$, Tao Tiann, ${ }^{1, *}$ Hai-song Tan $^{3, *}$, Ying Chen ${ }^{2}$, \\ Guang-lei Qiao ${ }^{2}$, Jun Han', Sheng-yu Huang ${ }^{6}$, Yuan Yang ${ }^{1}$, Shuai Li ${ }^{4}$, Zhen-guang \\ Wang ${ }^{1}$, Rong Gao ${ }^{5}$, Hao Ren ${ }^{5}$, Hao Xing ${ }^{1}$, Jun-sheng Ni ${ }^{1}$, Lin-Hui Wang ${ }^{3}$, Li-jun Ma ${ }^{2}$, \\ Wei-ping Zhou ${ }^{1}$ \\ ${ }^{1}$ The Third Department of Hepatic Surgery, Eastern Hepatobiliary Surgery Hospital, Second Military Medical University, \\ Shanghai 200433, China \\ ${ }^{2}$ Department of Oncology, Shanghai Tongren Hospital, Shanghai Jiaotong University, Shanghai 200336, China \\ ${ }^{3}$ Department of Urology, Changzheng Hospital, Second Military Medical University, Shanghai 200003, China \\ ${ }^{4}$ Department of Computer Science, Rensselaer Polytechnic Institute, Troy, NY, 12180, United States of America \\ ${ }^{5}$ Department of Microbiology, Shanghai Key Laboratory of Medical Biodefense, Second Military Medical University, Shanghai \\ 200433, China \\ ${ }^{6}$ The Fourth Department of Hepatic Surgery, Eastern Hepatobiliary Surgery Hospital, Second Military Medical University, \\ Shanghai 200433, China \\ *These authors have contributed equally to this work
}

Correspondence to: Wei-ping Zhou, email: ehphwp@126.com

Li-jun Ma, email: lijun.ma@shtrhospital.com

Lin-Hui Wang, email: wanglinhuicz@163.com

Keywords: CBX6, S100A9, hepatocellular carcinoma, proliferation, biomarker

Received: December 06, 2016

Accepted: January 09, 2017

Published: January 20, 2017

\section{ABSTRACT}

Aberrant chromobox (CBX) family protein expression has been reported in a variety of human malignancies. However, the role of CBX6 in hepatocellular carcinoma (HCC) progression and patient prognosis remains unknown. In this study, we found that CBX6 was frequently up-regulated in HCC clinical samples and HCC cell lines and that CBX6 expression was significantly correlated with larger tumor sizes ( $\geq 5 \mathrm{~cm}, p=0.011$ ) and multiple tumors $(n \geq 2, p=0.018)$. Survival analyses indicated that patients with higher CBX6 expression levels had significantly shorter recurrence-free survival (RFS) and overall survival (OS) than patients with lower CBX6 expression levels, and multivariate analyses confirmed that increased CBX6 expression was an independent unfavorable prognostic factor for HCC patients. Functional study demonstrated that CBX6 profoundly promoted HCC cell growth both in vitro and in vivo, and mechanistic investigation revealed that the S100A9/NF-KB/MAPK pathway was essential for mediating CBX6 function. In conclusion, our results represent the first evidence that CBX6 contributes to tumor progression and indicate that the protein may serve as a novel prognostic biomarker for HCC and as a therapeutic target in the treatment of the disease.

\section{INTRODUCTION}

Primary liver cancer is the fifth-most common cancer worldwide and the third-most common cause of cancer mortality. Hepatocellular carcinoma (HCC) accounts for nearly $90 \%$ of primary liver cancers $[1,2]$. HCC always develops in the background of chronic hepatitis or cirrhosis. Inflammatory cells invade the liver as a result of the destruction of large numbers of hepatocytes, leading to the deposition of connective tissue in chronic liver disease [3-5]. Although multiple tumor suppressor genes and oncogenes that participate in $\mathrm{HCC}$ development and progression have been identified [6,7], our knowledge of the cellular and molecular pathways underlying HCC progression remains limited.

The chromobox (CBX) protein family comprises chromodomain-containing proteins involved in regulating gene expression, cell self-renewal and differentiation [8-11]. The overall structures of CBX proteins are similar, and these 
proteins direct the localization of their respective complexes through specific recognition of distinct but sequencerelated repressive marks on histone $\mathrm{H} 3$ trimethylated at Lys-9 (H3K9me3) and Lys-27 (H3K27me3) [12-16]. The detailed functions of each protein remain unclear; however, these proteins play important roles in disease progression. $\mathrm{CBX} 1,3$, and 5 are associated with gene repression in heterochromatin, and $\mathrm{CBX} 7$ and 8 are key regulators of developmental genes targeted by H3K27me3. CBX2, 4, and 6 also perform this function but have weaker effects than their counterparts [9-11, 16]. CBX6 shows a high degree of conservation, but its chromodomains display significant differences from those of other proteins with respect to their histone peptide binding preferences. Previous studies have reported that CBX6 may be a reader of different, as-yet undetermined trimethyllysine sites and may be capable of binding to RNA $[9,15,17,18]$. However, the role of CBX6 in HCC development and progression has not been explored.

In the present study, we demonstrated that CBX6 expression is increased in HCC cells and tumor tissues and that increased $\mathrm{CBX} 6$ expression is associated with poor outcomes in a large $\mathrm{HCC}$ cohort. Additionally, the results of our univariate and multivariate analyses indicated that CBX6 was an independent prognostic factor for poor survival and HCC recurrence after surgery. Moreover, CBX6 up-regulation dramatically promoted HCC proliferation both in vitro and in vivo, while CBX6 downregulation dramatically attenuated HCC proliferation both in vitro and in vivo. Regarding the mechanism underlying the involvement of $\mathrm{CBX} 6$ in $\mathrm{HCC}$, gene microarray analysis of a CBX6-knockdown cell line revealed that S100A9 expression was low, suggesting that CBX6 plays an important role in HCC progression through S100A9/ $\mathrm{NF}-\kappa \mathrm{B} / \mathrm{MAPK}$ signaling and may be useful as a future HCC therapy.

\section{RESULTS}

\section{CBX6 expression is frequently increased in human HCC tissues}

To determine the significance of CBX6 expression in $\mathrm{HCC}$, we first detected CBX6 mRNA and protein expression levels in a normal human liver cell line (THLE3) and several human HCC cell lines (MHCC-97L, Huh7, HepG2, SMMC7721, HCCLM3, and MHCC-97H). Realtime quantitative polymerase chain reaction (qRT-PCR) and western blot analyses revealed that CBX6 mRNA and protein expression levels were both markedly increased in all six HCC cell lines compared to the THLE-3 cell line (Figure 1A).Then, we detected CBX6 mRNA expression in 50 pairs of primary HCC tissue samples and corresponding adjacent nontumor tissue samples. Our qPCR results showed that relative CBX6 mRNA expression levels were significantly higher in the tumor tissue samples than the adjacent non-tumor tissue samples, as $84 \%(42 / 50)$ of the HCC tissue specimens showed a higher CBX6 mRNA expression level than their matched non-tumor counterparts (Figure 1B). Similar results were observed in the western blot analysis (Figure 1C). To determine the protein expression patterns of CBX6 in the HCC clinical samples, we next performed immunohistochemistry (IHC) forCBX6 expression using HCC tissue microarrays (TMAs) containing 313 HCC samples and paired peritumor samples. As shown in Figure 1D, the staining intensity of the CBX6 protein in the tumor group was stronger than that in the peri-tumor group. Representative IHC staining results are shown in Figure 1E. We also detected the expression of other $\mathrm{CBX}$ family proteins in 50 pairs of $\mathrm{HCC}$ tissues and adjacent non-tumor tissues, and no up-regulations of CBX1-5,CBX 7, or CBX 8 expression were observed in the HCC tissue samples (Supplementary Figure $1 \mathrm{~A}-1 \mathrm{G}$ ). Our results revealed that $\mathrm{CBX} 6$ expression levels were frequently increased in $\mathrm{HCC}$, implying that the protein plays an oncogenic role in the disease.

\section{Association between CBX6expression and HCC patient clinicopathologic characteristics}

To further investigate the clinical significance of CBX6 expression in HCC development and progression, we divided all $313 \mathrm{HCC}$ patients into the following 2 groups based on their overall CBX6 expression levels: a high CBX6 expression group $(n=157)$ and a low CBX6 expression group $(n=156)$. We found that high CBX6expression was more frequent in HCC patients with larger tumor sizes ( $\geq 5 \mathrm{~cm}, p=0.011)$ and multiple tumors $(n \geq 2, p=0.018$ ) (Table 1$)$ than in HCC patients with smaller tumor sizes and single tumors.

\section{Relationship between CBX6 expression and HCC patient prognosis}

We analyzed the association between CBX6 protein levels and HCC patient prognosis after hepatectomy. We found that the high CBX6expression group had significantly poorer recurrence-free survival (RFS) $(p=0.006$, Figure 2A) and poorer overall survival (OS) $(p=0.001$, Figure $2 \mathrm{~B})$ than the low CBX6 expression group. Subgroup analysis revealed that among patients with a tumor size $<5 \mathrm{~cm}$ (140 patients), differences in RFS and OS still existed between the high and low CBX6 expression groups ( $p<0.001$; respectively, Figure $2 \mathrm{C}$ and 2D). Further analysis revealed that among AFCP-negative patients (115 patients), the high CBX6 expression group had significantly poorer RFS ( $p=0.021$, Figure 2E) and poorer OS than the low CBX6 expression group ( $p=0.018$, Figure $2 \mathrm{~F}$ ). Univariate analysis showed that of the clinicopathological characteristics analyzed herein, CBX6 expression levels, tumor size, vascular invasion, and the presence of AFP, $\mathrm{HBsAg}$, or $\mathrm{HBeAg}$ were correlated with RFS, and CBX6 expression levels, 
Table1: Clinical characteristics of 313 HCC patients according to their CBX6 expression levels

\begin{tabular}{|c|c|c|c|c|}
\hline \multirow{2}{*}{ Feature } & \multicolumn{2}{|c|}{ CBX6 } & \multirow{2}{*}{$\chi^{2}$} & \multirow{2}{*}{$p$-value } \\
\hline & High & Low & & \\
\hline \multicolumn{5}{|l|}{ All cases } \\
\hline Age, y & & & 0.026 & 0.872 \\
\hline$\geq 55$ & 58 & 59 & & \\
\hline$<55$ & 99 & 97 & & \\
\hline Gender & & & 0.000 & 0.984 \\
\hline Male & 139 & 138 & & \\
\hline Female & 18 & 18 & & \\
\hline Hepatitis B ag & & & 0.739 & 0.390 \\
\hline Positive & 139 & 133 & & \\
\hline Negative & 18 & 23 & & \\
\hline Hepatitis E ag & & & 0.643 & 0.422 \\
\hline Positive & 36 & 30 & & \\
\hline Negative & 121 & 126 & & \\
\hline AFP, $\mu \mathrm{g} / \mathrm{L}$ & & & 0.006 & 0.941 \\
\hline Positive & 99 & 99 & & \\
\hline Negative & 58 & 57 & & \\
\hline Tumor size, cm & & & 6.512 & 0.011 \\
\hline$\geq 5$ & 98 & 75 & & \\
\hline$<5$ & 59 & 81 & & \\
\hline Tumor number & & & 5.608 & 0.018 \\
\hline Single & 117 & 133 & & \\
\hline Multiple & 40 & 23 & & \\
\hline Vascular invasion & & & 0.729 & 0.393 \\
\hline Present & 98 & 90 & & \\
\hline Absent & 59 & 66 & & \\
\hline Tumor differentiation & & & 1.145 & 0.285 \\
\hline $\mathrm{I}-\mathrm{II}$ & 20 & 14 & & \\
\hline III-IV & 137 & 142 & & \\
\hline
\end{tabular}

Values in red are statistically significant $(p<0.05)$.

AFP, alpha-fetoprotein; HBsAg, hepatitis B surface antigen; HBeAg, hepatitis Be antigen; negative: $<20 \mathrm{ng} / \mathrm{ml}$, positive: $\geq 20 \mathrm{ng} / \mathrm{ml}$.

aPatients whose tumor tissue immunohistochemistry score was $>1$ constituted the high-expression group, and the remaining patients constituted the low-expression group.

tumor size, the presence of HBsAg, and the presence of HBeAg were correlated with OS (Supplementary Table 1). Furthermore, multivariate Cox regression analysis showed that CBX6 expression levels, vascular invasion, the presence of $\mathrm{HBeAg}$, and the presence of $\mathrm{HBsAg}$ were independent risk factors for $\mathrm{HCC}$ recurrence and that $\mathrm{CBX} 6$ expression levels, the presence of $\mathrm{HBeAg}$, and the presence of HBsAg were independent risk factors for OS in HCC patients (Table 2). Taken together, these data indicate that CBX6expression levels can be used as an independent factor for predicting the prognosis of HCC.

\section{CBX6 promotes HCC cell proliferation and HCC cell clonogenicity in vitro}

Given the significant correlation between CBX6expression levels and HCC clinical invasiveness, it is likely that CBX6 plays a positive role in cancer progression. To investigate the biological significance of CBX6 in vitro, we examined the effects of CBX6 loss-of-function and gain-of-function on cell phenotype. SMMC7721cells exhibited lower CBX6 expression and were thus selected to generate stable CBX6 over-expression cells (which 
we named SMMC-7721-CBX6), and HCCLM3 cells exhibited higher CBX6 expression and were thus selected to generate stable CBX6-knockdown cells (which we named HCCLM3-CBX6i).CBX6overexpression or knockdown efficiency was confirmed by qRT-PCR and western blotting (Supplementary Figure 2).Cell Counting Kit-8 (CCK8) was utilized to assess HCC cell proliferation in vitro. As shown in Figure 3A, CBX6 overexpression promoted SMMC-7721 cell proliferation, and the difference in cell growth between the knockdown and control cell lines continued to increase at subsequent time points. Conversely, HCC-LM3 cells with
CBX6 knockdown displayed notable reductions in their proliferation rate compared with their corresponding control cells (Figure 3B). To evaluate the long-term effects of CBX6 on cell proliferation, we performed colony-formation assays. As shown in Figure 3C and 3D, significantly more tumor colonies had formed in the CBX6-overexpression group than in the CBX6-knockdowngroup on day 14. We next examined the anti-apoptotic effects of CBX6 in HCC cells via flow cytometric analysis. Surprisingly, no significant differences in apoptosis were noted between the stable CBX6-overexpression cell line and its control cell line or
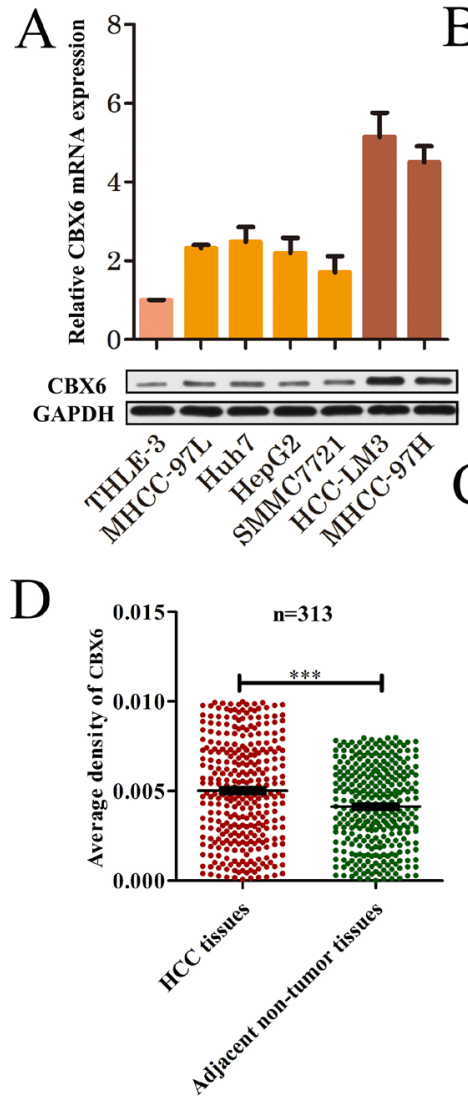

B
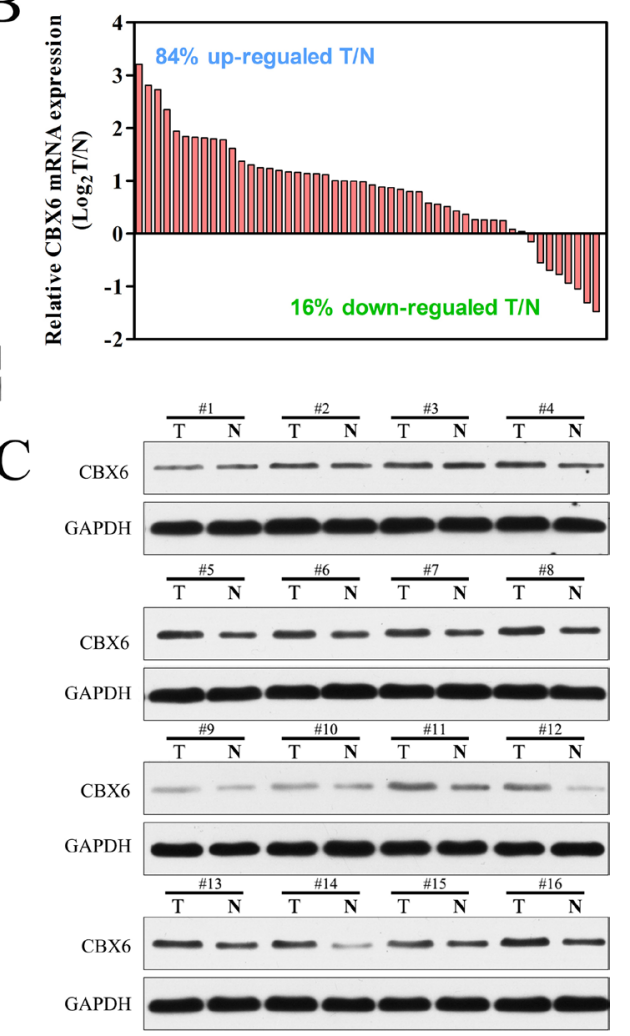

$\mathrm{E}$
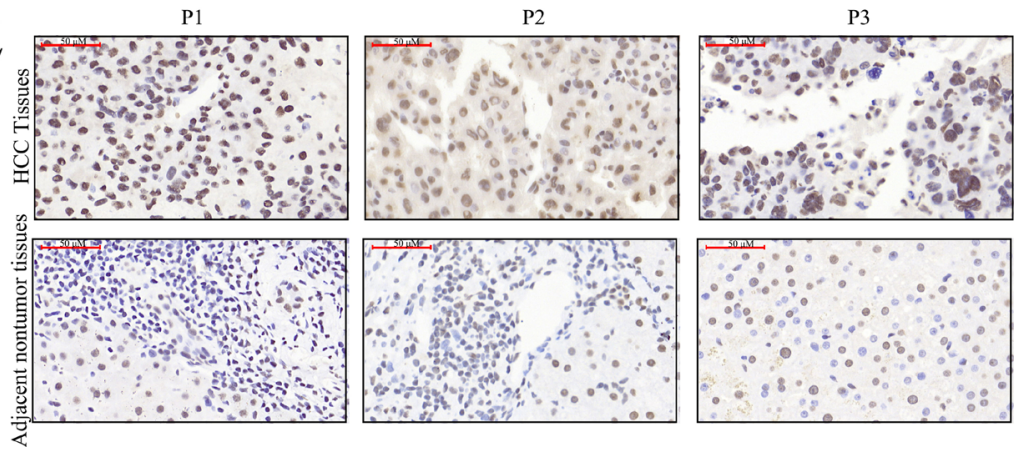

Figure 1: CBX6 expression is frequently increased in human HCC tissues. (A) Relative CBX6 mRNA and protein expression levels in the THLE-3 and HCC cell lines were determined by real-time qPCR and western blot assay. The gene expression results were normalized to GAPDH, which served as an internal control. (B) CBX6 mRNA expression levels in 50 pairs of HCC tissues and matched adjacent nontumor tissues were evaluated by qPCR. (C)Western blot analysis of CBX6 protein levels in tumor tissues and paired adjacent non-tumor tissues from 16 patients with HCC. (D)Relative IHC staining for CBX6 expression in HCC tissue samples and paired adjacent non-tumor tissue samples $(n=313)$.CBX6 expression was significantly increased in the tumor tissue samples compared with the corresponding adjacent non-tumor tissue samples. (E)Representative IHC image of tumor tissues and peri-tumor tissues $\left({ }^{*} p<0.05 ; * * p<0.01 ; * * * p<0.001\right)$. 
Table 2: Multivariable analysis of recurrence free survival (RFS) and overall survival (OS) in patients

\begin{tabular}{lcccccccc}
\hline \multirow{2}{*}{\multicolumn{1}{c}{ Variable }} & \multicolumn{4}{c}{ RFS } & \multicolumn{3}{c}{ OS } \\
\cline { 2 - 9 } & $\boldsymbol{P}$ & HR & $\mathbf{9 5 \%}$ CI & P & HR & 95\% CI \\
\hline HBsAg, positive vs. negative & 0.020 & 1.839 & 1.099 & 2.318 & 0.019 & 1.927 & 1.114 & 3.332 \\
HBeAg, positive vs. negative & 0.003 & 1.662 & 1.192 & 2.318 & 0.003 & 1.656 & 1.185 & 2.314 \\
Vascular invasion, present vs. absent & 0.004 & 1.580 & 1.160 & 2.152 & - & - & - & - \\
CBX6 expression, high vs. low & 0.018 & 1.423 & 1.061 & 1.908 & 0.003 & 1.591 & 1.175 & 2.153 \\
\hline
\end{tabular}

Abbreviation: HBsAg, hepatitis B surface antigen; HBeAg, hepatitis B e antigen.

between the CBX6-knockdown cell line and its control cell line following treatment with apoptosis-inducers A (Apopida) and B (Apobid) for $6 \mathrm{~h}$ (Supplementary Figure 3A). Apoptosis-associated protein expression levels in the SMMC-7721-CBX6 and HCC-LM3-CBX6i cell lines and their respective control cell lines were also evaluated by western blotting. Similar to the above results, no differences in apoptosis-associated protein levels were observed between the above cells and their respective control cells (Supplementary Figure 3B). These results indicate that CBX6has no anti-apoptotic effects in HCC cells. Taken together, our results indicate that $\mathrm{CBX} 6$ promotes $\mathrm{HCC}$ cell proliferation and $\mathrm{HCC}$ cell clonogenicity in vitro.

\section{CBX6 promotes HCC tumor growth in vivo}

To determine the effects of $\mathrm{CBX} 6$ on tumourigenesis in vivo, we subcutaneously injected SMMC-7721-NC cells or SMMC-7721-CBX6cells into nude mice for xenotransplantation. The results of this experiment showed that CBX6 overexpression evidently promotedtumor growth. As shown in Figure 4A, mice injected with SMMC-7721-CBX6 cells showed significantly increased tumor growth compared with mice injected with SMMC7721-NC cells. The results of our tumor volume and mass measurements indicated thatCBX6 overexpression significantly increased overall tumor growth (Figure 4B and $4 \mathrm{C}$ ). We performed similar assessments of xenograft tumor formation in nude mice using CBX6 knockdown cells and the appropriate control cells. As shown in Figure 4D-4F, CBX6 knockdown tumors (HCCLM3CBX6i) showed significantly decreased tumor growth compared with control tumors (HCCLM3-NC), based on our measurements of tumor volume and weight. We also measured the levels of two cellular proliferation antigens, Ki67 and PCNA, in tumor tissues from the xenografts via IHC and found that the expression levels of both of these antigens were significantly stronger in theSMMC-7721CBX6cell xenografts than in theSMMC-7721-NC cell xenografts (Supplementary Figure 4A).In contrast, the expression levels of Ki67 and PCNA were significantly lower in the HCCM3-CBX6i cell xenografts than in the HCCLM3-NCcell xenografts (Supplementary Figure 4B).
These results revealed that CBX6 was capable of promoting tumor growth in vivo and acted as an oncogene in HCC.

\section{CBX6 increased S100A9 expression in HCC}

To identify the genes that are potentially involved in CBX6-mediatedcell proliferation, we constructed gene microarrays. The gene expression data were obtained from three independent competitive hybridizations comparing PON3-knockdown HCCLM3 cells (HCCLM3-CBX6i) with control cells (HCCLM3-NC). A corrected $p$-value $<0.05$ and an absolute fold change $>1.2$ were the criteria used for identifying differentially expressed genes. A total of 240 genes were found to be differentially expressed. Of these, 115 genes were down-regulated, and 125 genes were up-regulated. We found that the expression of S100A9, one of the top ten down-regulated genes in HCCLM3-CBX6i cells, was dramatically induced by CBX6 (Figure 5A). Given that S100A9 functions in cell proliferation [19], we surmised that CBX6may promote proliferation by increasing S100A9 expression. The results of previous studies indicated that S100A9 regulated MAPK signaling and NF-Kb signaling [20]; therefore, we tested the phosphorylation of the MAPK and NF- $\mathrm{kB}$ signaling pathways by western blot analysis and enzyme activity assay. Western blot analysis revealed that the protein expression levels of phospho-ERK1/2 MAPK, phospho-p38 MAPK, phospho-p50 NF- $\kappa B$ and phospho-p65 NF- $\mathrm{KB}$ in the SMMC-7721-CBX6 cell line were higher than those of the same proteins in the SMMC-7721-NCcell line and that the protein expression levels of phospho-ERK1/2 MAPK, phospho-p38 MAPK, phospho-p50 NF- $\mathrm{KB}$ and phospho-p65 NF- $\mathrm{KB}$ in the HCCLM3-CBX6i cell line were lower than those of the same proteins in the HCCLM3-NC cell line (Figure 5B). Enzyme activity assay confirmed the above results (Figure 5C-5F). To confirm that S100A9 is regulated by CBX6 in human HCC tissues, we measured S100A9 mRNA levels in 50 pairs of HCC tissues and matched nontumor tissues, as shown in Figure 1B, and found that S100A9 mRNA levels were significantly higher in the HCC tissue samples than in the primary nontumor tissue 

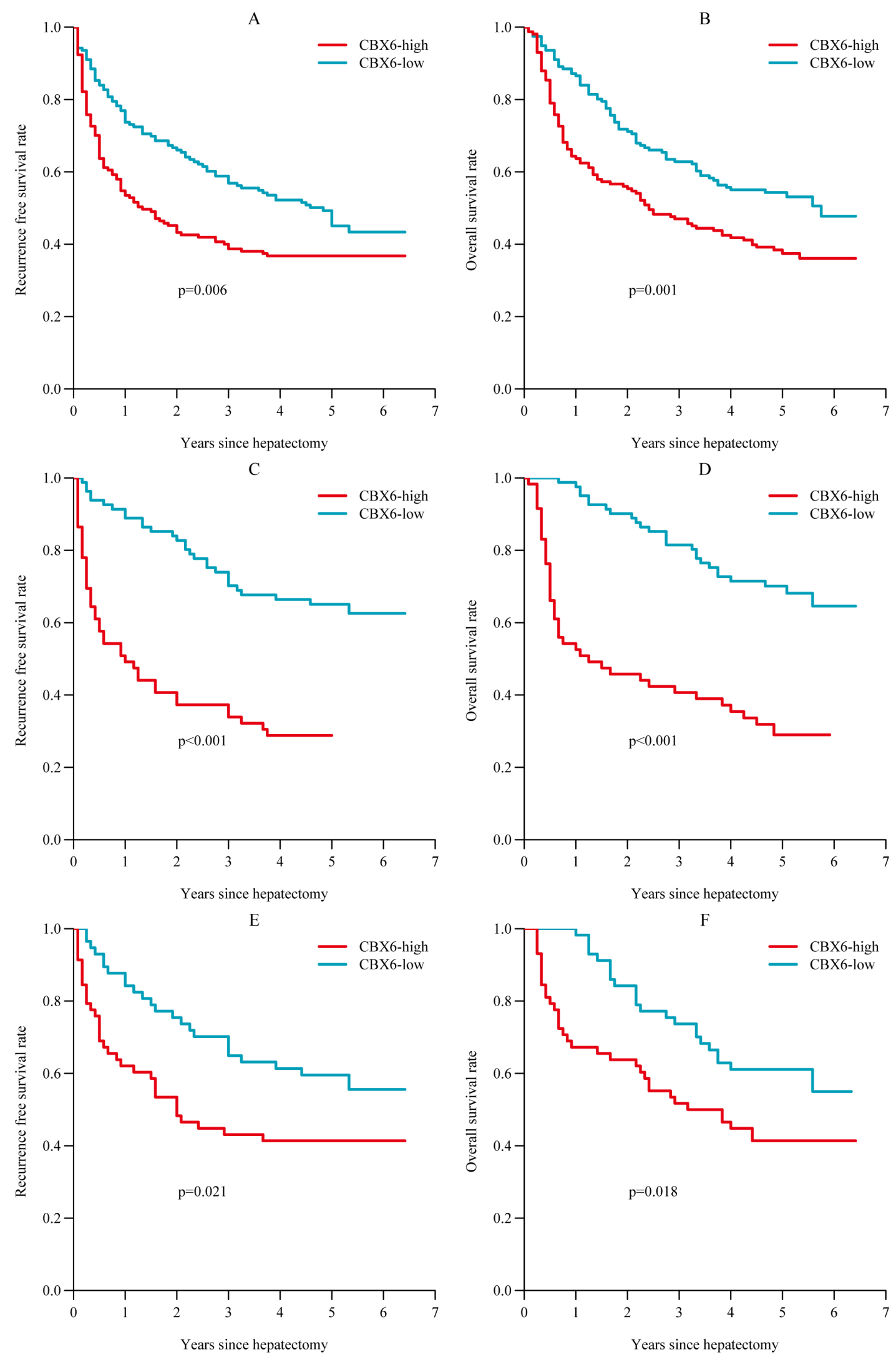

Figure 2: Relationship between CBX6 expression and HCC patient prognosis. (A and B) Postoperative RFS and OS among all HCC patients. (C and D) Postoperative RFS and OS among HCC patients with a tumor size $<5 \mathrm{~cm}$. (E and $\mathbf{F}$ ) Postoperative RFS and OS among HCC patients with an AFP $<20 \mathrm{ng} / \mathrm{ul}$. Statistical significance was assessed by two-sided log-rank tests $\left({ }^{*} p<0.05 ; * * p<0.01 ; * * * p<0.001\right)$. 
samples (Figure 5G). Importantly, S100A9 mRNA levels were correlated withCBX6 transcript levels in the HCC tissue samples (Figure 5H). These clinical data indicate that S100A9 may be associated with HCC cell metastasis and were consistent with our hypothesis regarding the role of CBX6 in S100A9 regulation.

\section{S100A9 plays a critical role in mediating CBX6 function}

After confirming that CBX6 can regulate S100A9 expression in HCC, we explored whether S100A9 can mediate the biological function of CBX6 in HCC cells further. We found that S100A9 lentivirus transection significantly increased S100A9 expression levels in HCCLM3-CBX6i cells ( $p<0.01$, Supplementary Figure 5A), while S100A9 siRNA significantly reduced S100A9 expression levels in SMMC-772-CBX6 cells ( $p<0.01$, Supplemental Figure 5B). Functionally, S100A9 knockdown abrogated the positive effects ofCBX6on SMMC-7721-CBX6 cell proliferation (Figure 5I).However, S100A9 overexpression abrogated

A

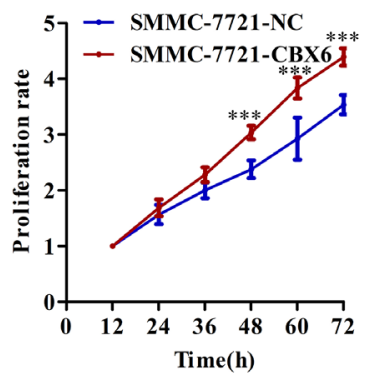

C
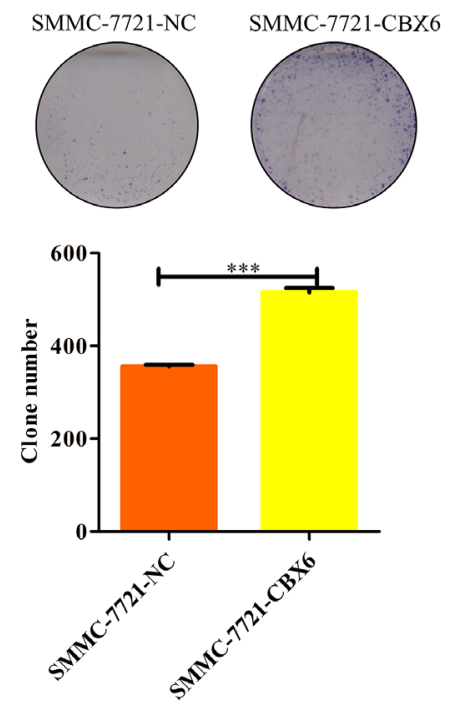

the inhibitory effects of CBX6 knockdown on HCCLM3CBX6i cell proliferation (Figure 5J). These results suggest that S100A9 is not only a downstream target of CBX6 but also a functional mediator of the effects of CBX6 in HCC.

\section{DISCUSSION}

The histopathological and molecular features that lead to HCC initiation and progression are still poorly understood [21]. Viral infection, metabolic alterations leading to chronic inflammation and epigenetic and genetic changes cooperate in cancer development [22]. Hepatocarcinogenesis is a complex process characterized by increases in the expression of several factors that influence the survival of cancer cells by suppressing apoptosis and regulating cell cycle [23-25]. Increases in the incidence of HCC have led to the performance of intense research intended to elucidate the detailed mechanisms underlying HCC development.

In the present study, we showed that CBX6 expression in HCC tissues was significantly up-regulated

B

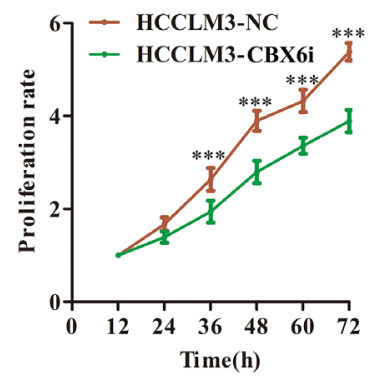

$\mathrm{D}$
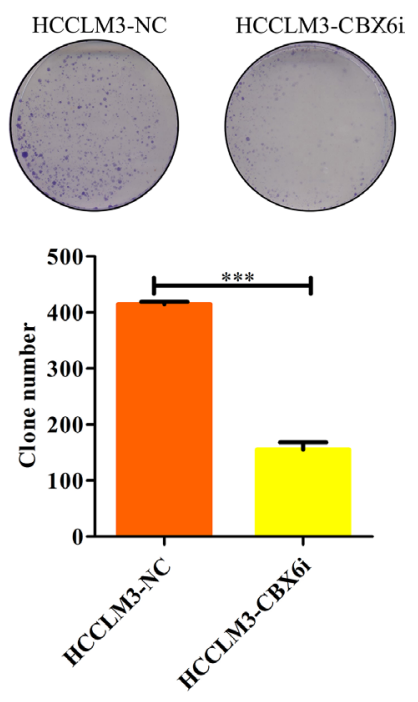

Figure 3: CBX6 promotes HCC cell proliferation and HCC cell clonogenicity in vitro. (A and B) Cell proliferation was determined by CCK-8 assay, and growth curves were generated by reading the absorbance values at different time points. Growth curves of (A) CXB6-overexpression cells and (B) CBX6-knockdown cells and their respective controls are shown. (C and D) Representative results of the colony formation assays of (C) CBX6-overexpression cells, (D) CBX6 knockdown cells and their respective controls. ${ }^{*} p<0.05$; $* * p<0.01 ; * * * p<0.001$ compared with the respective controls. Significance was determined from three independent experiments and assessed by Student's $t$-test. Data are shown as the mean $\pm \mathrm{SD}$. 
compared with that in adjacent normal liver tissues. The results of our IHC analysis of TMA slides from a cohort of 313 randomly selected HCC patients and our KaplanMeier analysis showed that up-regulated CBX6 expression was predictive of poor HCC patient survival. These results suggest that CBX6 may play an important role in $\mathrm{HCC}$ progression. The results of a previous study indicated that patients with a tumor size $<5 \mathrm{~cm}$ who are AFP negative are generally considered to have a better prognosis than patients with a tumor size $>5 \mathrm{~cm}$ who are AFP positive [26-29]; however, many of these patients still experience early disease recurrence and poor OS. Therefore, a precise biomarker for $\mathrm{HCC}$ is needed to predict the prognoses of patients with a smaller tumor size who are AFP negative. In our study, we found that within these populations, the high-expression group still had significantly poorer RFS than the low-expression group. Thus, the findings of the present study suggest that measuring CBX6protein levels may enable clinicians to identify patients with early stage disease who face a worse prognosis than other patients with early stage disease.CBX6 may thus have prognostic value because it may allow clinicians to distinguish between early stage patients with a high risk of recurrence and early stage patients with a low risk of recurrence. In the former group of patients, close follow-up and appropriate adjuvant therapies should be recommended to prolong survival.
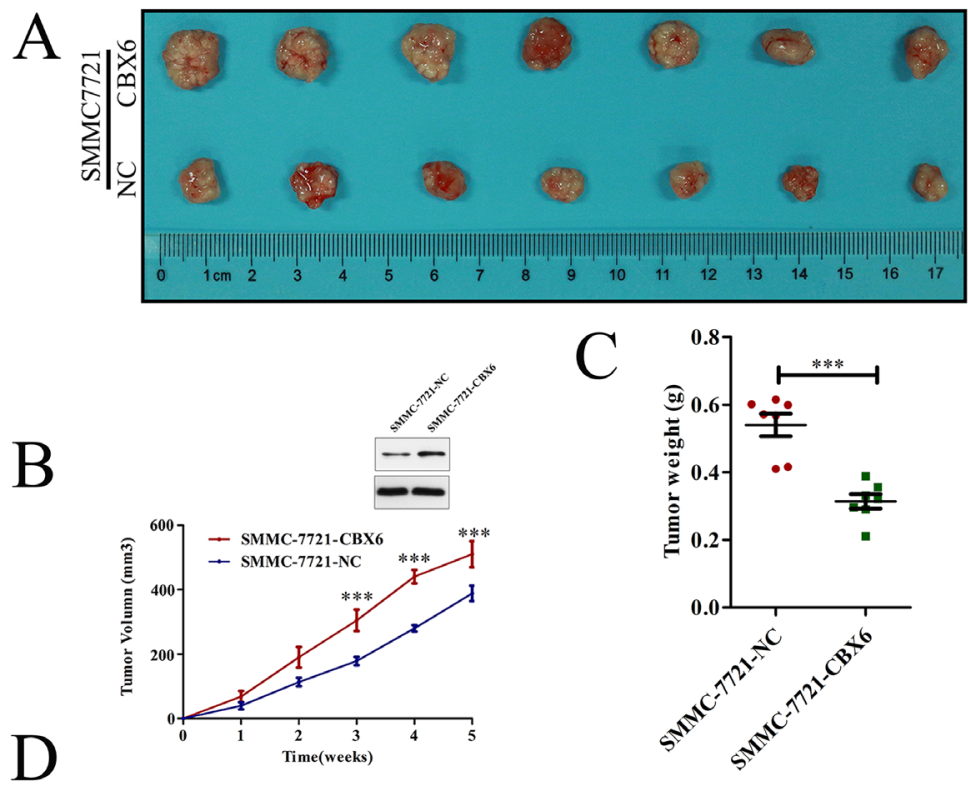

$\mathrm{D}$
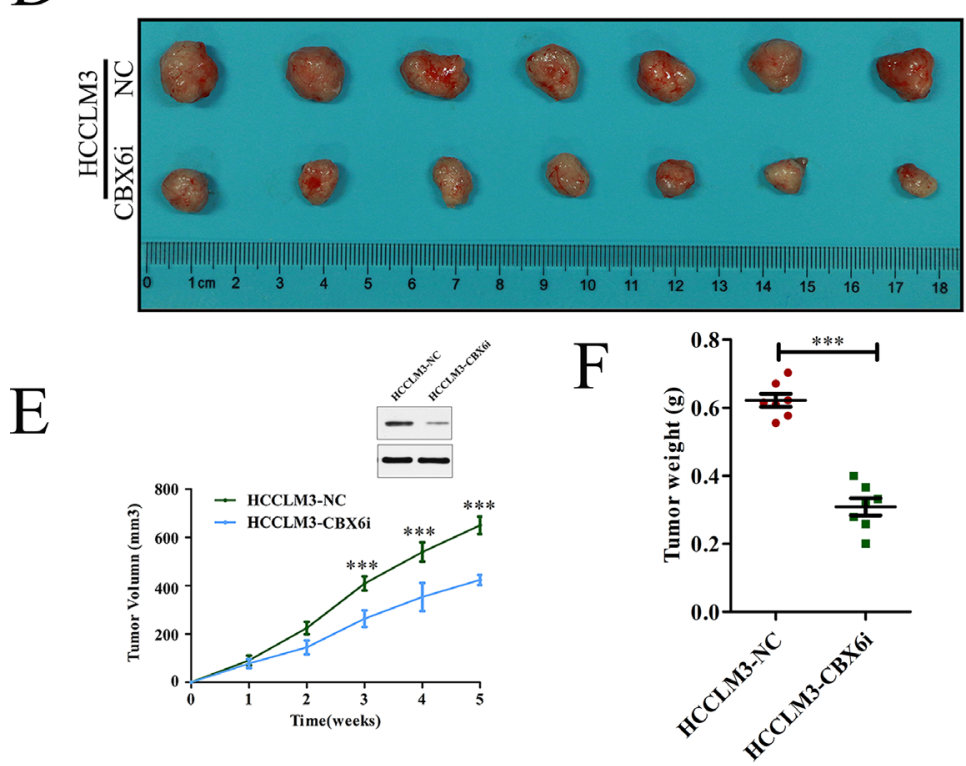

Figure 4:CBX6 promotes HCC tumor growth in vivo. Mice received subcutaneous injections of $1 \times 10^{7}(\mathrm{~A}-\mathrm{C}) \mathrm{SMMC}-7721-\mathrm{CBX} 6$ and SMMC-772-NC cells and (D-F) HCCLM3-CBX6i and HCCLM3-NC cells. (A and D) The mice and their tumors were evaluated five weeks post-injection, and representative images are shown. (B and E) Tumor growth curves and (C and F) tumor weights for the fiveweek study period are shown. Data are shown as the mean \pm SEM. Statistically significant differences were identified by Student's $t$-tests. ${ }^{*} p<0.05 ; * *<0.01 ; * * * p<0.001$ compared with the appropriate controls. 
By analyzing the association between CBX6 protein levels and clinicopathological characteristics using TMA analysis, we found that high CBX6expression was more frequent in $\mathrm{HCC}$ patients with a larger tumor size $(\geq 5 \mathrm{~cm}$, $p=0.011)$ and multiple tumors $(n \geq 2, p=0.018)$ than in patients with a smaller tumor size and single tumors, indicating that CBX6expression is closely correlated with tumor progression. We next explored the possible function of CBX6 in HCC progression. The results of this experiment showed that CBX6 promoted HCC cell proliferation in vitro and in vivo. Taken together, our data indicate that CBX6 plays an important role in $\mathrm{HCC}$ progression and suggest that $\mathrm{CBX}$ may be useful as a novel biomarker that can predict $\mathrm{HCC}$ prognosis. Our results also indicate that $\mathrm{CBX} 6$ may be a therapeutic target in the treatment of $\mathrm{HCC}$; however, the molecular mechanisms underlying the involvement of CBX6 in HCC proliferation remain to be elucidated.
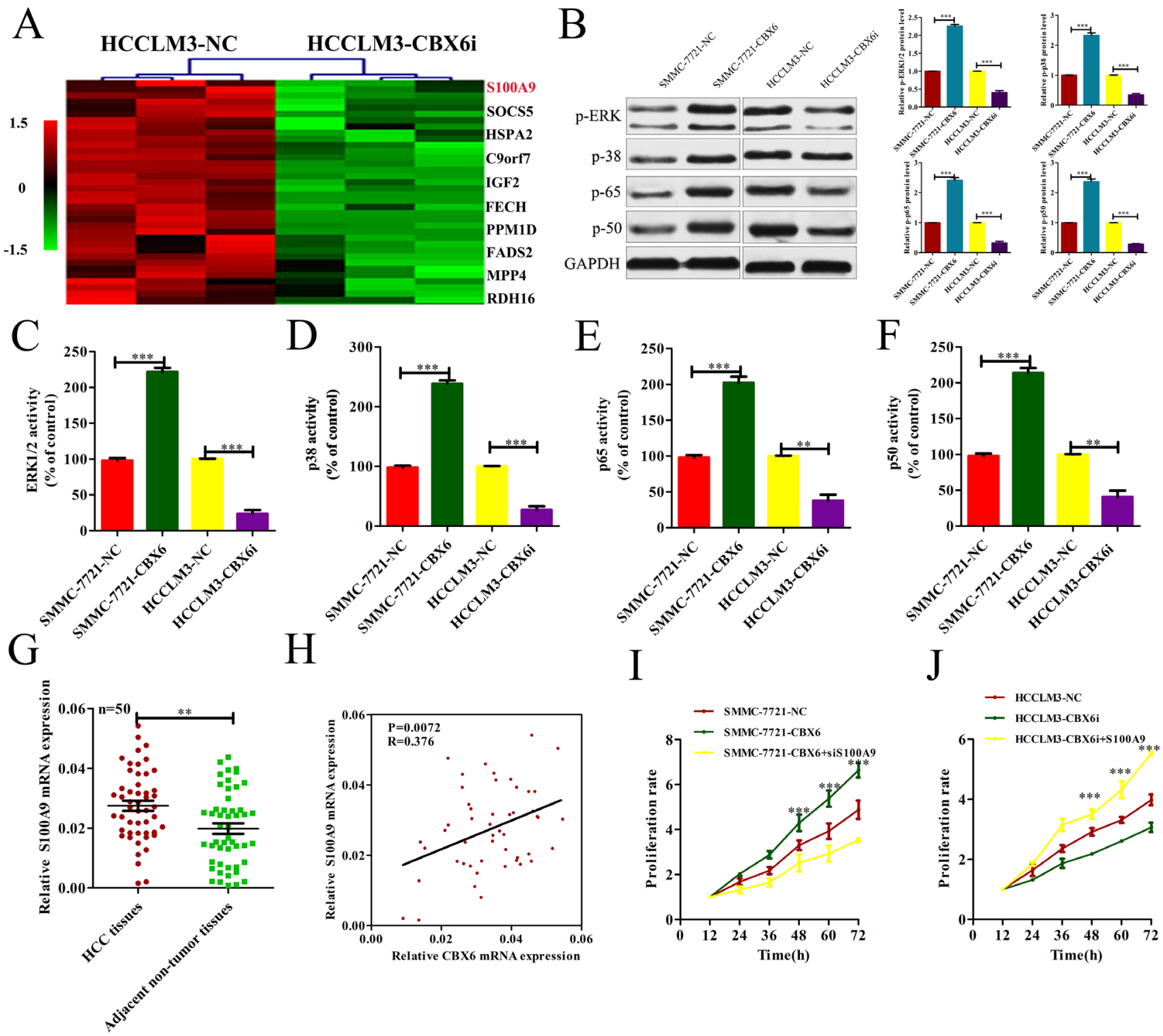

Figure 5: CBX6 increased S100A9 expression, and S100A9 plays a critical role in mediating CBX6 function in HCC. (A) Genes differentially expressed between HCCLM3-CBX6i cells and control cells. The top 10 down-regulated genes are listed on the

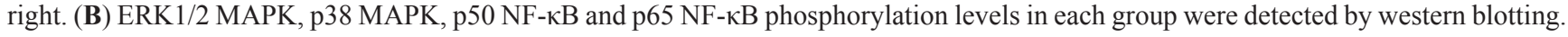

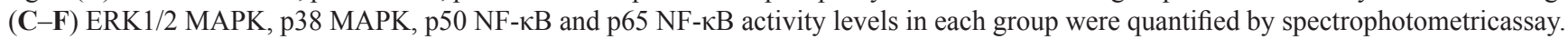
(G) S100A9 mRNA levels in primary HCC tissue samples and adjacentnontumor tissuesamples from the same set of patients as those described in Figure 1B were measured by qRT-PCR. (H) The correlation between CBX6 transcript levels and S100A9 mRNA levels was measured in the same set of HCC tissues as those described in (G). (I) CCK8 assay for SMMC7721 cells overexpressing CBX6 and SMMC7721 cells overexpressing CBX6 that were transfected with a S100A9-knockdown vector or a control vector. (J) CCK8 assay for HCCLM3 cells treated with CBX6 knockdown and HCCLM3 cells treated with CBX6 knockdown that were transfected with an S100A9 vector or a control vector. $* p<0.05 ; * * p<0.01 ; * * p<0.001$. 
To identify genes that are potentially involved in CBX6-mediated cell proliferation, we constructed gene microarrays. We found that the expression of S100A9, one of the top ten down-regulated genes, was significantly decreased. S100A9 belongs to the S100 gene cluster and was originally discovered inhuman granulocytes and macrophages [30-33].S100A9 has been shown to be associated with myelomonocytic cell differentiation [34], andit is well known that S100A9 deregulation is associated with several common human malignancies and induces neutrophil adhesion to fibronectin $[35,36]$.

In a previous study, S100A9 was found to mediate proliferative signals and enhance the MAPK or NF- $\mathrm{BB}$ signaling pathway [37]. Aberrant activation of both the MAPK and the NF- $\kappa \mathrm{B}$ signaling pathway has critical effects on tumor growth [38-41], according to the results previous studies [42]. We observed that CBX6suppression caused decreases in ERK1/2 phosphorylation and MAPK-p38, NF- $\kappa$ B-p50, and NF- $\kappa$ B-p65 phosphorylation and thatCBX6overexpression increased ERK1/2 phosphorylation and MAPK-p38,NF- $\mathrm{B}-\mathrm{p} 50$, and NFkB-p65 phosphorylation, indicating that CBX6may mediate tumor progression in HCC cells by promoting cellular proliferation, which is linked to the MAPK and $\mathrm{NF}-\kappa \mathrm{B}$ signaling pathways. We also found that knocking downS100A9using siRNA decreased HCC cell proliferation and reversed the $\mathrm{HCC}$ cell proliferation enhancements induced by CBX6 and that S100A9 overexpression increased $\mathrm{HCC}$ proliferation and reversed the decrease in proliferation induced by CBX6 knockdown. All of these results suggest that $\mathrm{CBX} 6$ promotescell proliferation, partly through S100A9 up-regulation. However, as described above, as information regarding the precise mechanism by which CBX6 increases S100A9 expression is lacking, the relationship between $\mathrm{CBX} 6$ and S100A9 is still unclear and requires further investigation.

In conclusion, we investigated the expression of CBX6 and its value as a prognostic biomarker for HCC. We also explored the biological function of CBX6 in $\mathrm{HCC}$ and observed that CBX6 up-regulation is a predictor of high risks of metastasis and recurrence in HCC patients. Highrisk patients should be closely monitored and should receive appropriate adjuvant therapies so that their prognoses can be improved. We also found that CBX6 promoted HCC proliferation by elevating S100A9 expression. These findings suggest that CBX6 plays an important role in HCC development and progression and may be a useful therapeutic target in the treatment of the disease.

\section{MATERIALS AND METHODS}

\section{Patient characteristics and tissue specimens}

All the patients enrolled in our study were infected with HBV. We obtained 50 HCC tissue samples and an equal number of paired adjacent non-tumor tissue samples for qRT-PCR analysis. A TMA containing 313 pairs of HCC tissues and adjacent non-tumor tissues was constructed for IHC testing. HCC differentiation was defined according to the Edmondson-Steiner classification. Micrometastaes were defined as tumors adjacent to the border of the primary tumor that could be observed only under a microscope. All tissue samples were randomly collected at the Eastern Hepatobiliary Surgery Hospital (Shanghai, China) from September 2005 to March 2012 and were stored at $-80^{\circ} \mathrm{C}$ until needed for further use. Two pathologists re-evaluated the tissues independently. The study was approved by the ethics committee of the Eastern Hepatobiliary Surgery Hospital, and written informed consent was obtained from all the study participants, in accordance with the policies of the ethics committee. RFS was defined as the period extending from the date of tumor resection to the date of tumor recurrence detection, the date of death from a non-HCC cause, or date of the last follow-up visit, and OS was defined as the length of time between the date of surgery and either the date of death of the patient or the date of the last follow-up visit. Information that could reveal the identities of the patients was excluded from this report.

\section{Cell culture}

The indicated hepatocellular carcinoma cell lines (SMMC-7721, HCC-LM3, Huh7, HepG2, MHCC97 L, MHCC-97H) and normal liver cell line (THLE-3) were obtained from the China Center for Type Culture Collection (Wuhan, China) and were authenticated by the provider using DNA-fingerprinting or isoenzyme analysis. All cell lines were maintained in DMEM (HyClone, UT, USA) containing $10 \%$ fetal bovine serum and $1 \%$ penicillin/streptomycin (GibcoBRL, MD, USA) at $37^{\circ} \mathrm{C}$ in a humidified atmosphere of 5\% CO2.

\section{RNA extraction, cDNA preparation, and qRT- PCR analysis}

Total RNA was extracted from frozen tissues or cells using Trizol reagent (Takara, Dalian, China), according to the manufacturer's instructions. Reverse-transcription was performed using 1-2 $\mu \mathrm{g}$ of total RNA, random primers, and the M-MLV Reverse-transcriptase Kit (Invitrogen, CA, USA), and qRT-PCRwas performed using SYBR Green Master Mix (Takara, China) in a StepOne Plus system (Applied Biosystems, CA, USA). $\beta$-actin served as an endogenous control. The qRT-PCR primers are listed in Supplementary Table 2. Relative RNA expression levels were calculated using the comparative $\mathrm{Ct}$ method.

\section{IHC tissue sections and TMA analyses}

Paraffin-embedded tissue sections and TMAs underwent IHC analyses. Briefly, the slides were probed 
with primary antibodies specific for the following proteins CBX6, PCNA, and Ki67, and then the slides were treated with anti-rabbit or anti-mouse horseradish peroxidase-conjugated secondary antibodies (Santa Cruz Biotechnology). Finally, the slides were stained with diaminobenzidine (DAB) colorimetric reagent solution from Dako (Carpinteria, CA, USA) before undergoing hematoxylin counterstaining (Sigma Chemical Co). TMA analysis was performed by scanning the slides with an AperioScanScope GL, and AperioImageScope software (Aperio Technologies, Vista, CA, USA) was used to assess the scanned images by determining the percentages of positively stained cells and staining intensities. CBX6expression in all the clinical samples was quantified, and the tumor CBX1-8 expression level/peri-tumor CBX18 expression level ratio was calculated.

\section{Westernblot analysis}

Total cell and tissues lysates were prepared in $1 \times$ sodium dodecyl sulfate buffer. Identical quantities of proteins were separated by sodium dodecyl sulfate-polyacrylamide gel electrophoresis and transferred onto nitrocellulose filter membranes. The blots were incubated with antibodies specific for CBX6 (Abcam, CA, USA), S100A9 (Abcam, CA, USA), total-ERK1/2(Abcam, CA, USA), phosphoERK1/2 (Abcam, CA, USA),total-p38(Abcam, CA, USA), phospho-p38(Abcam, CA, USA), total-p65 (Abcam, CA, USA), phospho-p65 (Abcam, CA, USA), total-p50 (Abcam, CA, USA), phospho-p50 (Abcam, CA, USA), or GAPDH (Abcam, CA, USA), after which they were incubated with IRDye 800-conjugated goat anti-rabbit IgG and IRDye 700-conjugated goat anti-mouse IgG, and the signals were detected using an Odyssey infrared scanner (Li-Cor). GAPDH was used as a loading control for these experiments.

\section{Animal studies}

The animal studies were approved by the Institutional Animal Care and Use Committee of the Second Military Medical University, Shanghai, China. Male athymic BALB/c nude mice (4-5 weeks old) were used for these experiments and received humane care throughout the experimental period. Equal numbers $\left(1 \times 10^{7}\right)$ of transduced HCC-LM3-CBX6i or SMMC-7721-CBX6 cells and the appropriate control cells were simultaneously injected into the bilateral armpits of each mouse. Tumor length (L) and width (W) were measured weekly after injections, and tumor growth curves were plotted using tumor volumes $\left(\mathrm{V}=0.5 \times \mathrm{L} \times \mathrm{W}^{2}\right)$ at each time point. All mice were sacrificed five weeks following injection.

\section{CCK8 assay and colony-formation assay}

Approximately $1000 \mathrm{HCC}$ cells were plated in 96well plates, and cell viability was assessed in 3 replicates from 3 independent experiments by CCK8 (Dojindo, Kumamoto, Japan) every 12 hours. Cell proliferation curves were plotted using the absorbance values at each time point.

For colony-formation assay, the cells were seeded in 6-well plates at a density of 1000 cells per well. After 2 weeks of growth, the surviving colonies were fixed with $4 \%$ paraformaldehyde, stained with $0.1 \%$ crystal violet, and counted.

\section{Apoptosis analyses}

The cells were pretreated with apoptosis-inducers A (Apopida) and B (Apobid) (1:1,000, Beyotime, China) for $6 \mathrm{~h}$. The cells were subsequently trypsinized, rinsed with PBS, resuspended in $1 \times$ binding buffer, stained with annexin V-FITC/PI (BD Bioscience, CA, USA), and analyzed with a flow cytometer (BD Biosciences, CA, USA).

\section{Enzyme activity assay}

Enzyme activity assay (Genmed Scientifics Inc, Shanghai,China) for ERK1/2, p38, p65, and p50 was used to measure the intracellular enzyme activity of the above proteins. Fifty micrograms of protein samples were added to a 96-well-plate, and the corresponding enzyme activity levels were determined using an enzymelabeling instrument at different wavelengths, according to the manufacturer's instructions. The values are presented as a percentage (\%) of the control.

\section{Gene expression profiling and analysis}

Total RNA was extracted using TRIzol Reagent (Life Technologies, Carlsbad, CA, USA), according to the manufacturer's instructions, and the RNA integrity number (RIN) was determined using an Agilent Bioanalyzer 2100 (Agilent Technologies, Santa Clara, CA, USA). Acceptable total RNA preparations were purified further using an RNeasy Mini Kit (QIAGEN, GmBH, Germany) and RNaseFree DNase (QIAGEN, GmBH, Germany). Total RNA was amplified and labeled using a Low-input Quick Amp Labeling Kit, One-Color (Agilent Technologies, Santa Clara, CA, USA), according to the manufacturer's instructions, and labeled cRNA was purified using an RNeasy Mini Kit (QIAGEN, GmBH, Germany). Each slide was hybridized with $1.65 \mu \mathrm{g}$ of Cy3-labeled cRNA in a hybridization oven using a Gene Expression Hybridization Kit (Agilent Technologies, Santa Clara, CA, USA), according to the manufacturer's instructions. After $17 \mathrm{~h}$ of hybridization, the slides were washed in staining dishes (Thermo Shandon, Waltham, MA, USA) using a Gene Expression Wash Buffer Kit (Agilent Technologies, Santa Clara, CA, USA), according to the manufacturer's instructions. The slides were scanned with an Agilent Microarray Scanner (Agilent Technologies, Santa Clara, CA, USA).The default settings 
were used. Data were extracted with Feature Extraction Software 10.7 (Agilent Technologies, Santa Clara, CA, USA) at the Biotechnology Corporation, Shanghai, PR China. The raw data were normalized using the Quantile algorithm in GeneSpring 11.0 (Agilent Technologies, Santa Clara, CA, USA), and we considered genes that were up- or down-regulated by more than 1.2-fold in the independent biological duplicates.

\section{Statistical analysis}

All statistical analyses were performed using SPSS version 17.0 and GraphPad Prism 5.0 software. $\chi 2$ test or Fisher's exact test was used for analysis of qualitative variables, and Student's $t$-test or the Mann-Whitney test was performed as appropriate for analysis of quantitative variables. Survival curves were calculated using the Kaplan-Meier method and were compared by a log-rank test. Cox's proportional hazards model was used to identify the independent factors for survival and recurrence, based on variables selected after the univariate analysis. Twotailed tests were performed to generate $p$-values and $p<0.05$ was considered statistically significant.

\section{CONFLICTS OF INTEREST}

The authors declare no conflicts of interest.

\section{GRANT SUPPORT}

This work was supported by the National Key Basic Research Program of China (2014CB542102); the Science Fund for Creative Research Groups, NSFC, China (81221061); the National Natural Science Foundation of China (81672335); the State Key Infectious Disease Project of China (2012ZX10002010); and the Shanghai New Excellent Youth Plan (XYQ2013074).

\section{REFERENCES}

1. El-Serag HB, Rudolph KL. Hepatocellular carcinoma: epidemiology and molecular carcinogenesis. Gastroenterology. 2007; 132:2557-76.

2. Torre LA, Bray F, Siegel RL, Ferlay J, Lortet-Tieulent J, Jemal A. Global cancer statistics, 2012. CA Cancer J Clin. 2015; 65:87-108.

3. El-Serag HB. Epidemiology of viral hepatitis and hepatocellular carcinoma. Gastroenterology. 2012; 142:1264-73.e1.

4. Gomaa AI, Khan SA, Toledano MB, Waked I, TaylorRobinson SD. Hepatocellular carcinoma: epidemiology, risk factors and pathogenesis. World J Gastroenterol. 2008; $14: 4300-8$.

5. Thorgeirsson SS, Grisham JW. Molecular pathogenesis of human hepatocellular carcinoma. Nat Genet. 2002; 31:339-46.
6. Hu TH, Wang CC, Huang CC, Chen CL, Hung CH, Chen $\mathrm{CH}$, Wang JH, Lu SN, Lee CM, Changchien CS, Tai MH. Down-regulation of tumor suppressor gene PTEN, overexpression of $\mathrm{p} 53$, plus high proliferating cell nuclear antigen index predict poor patient outcome of hepatocellular carcinoma after resection. Oncol Rep. 2007; 18:1417-26.

7. Ye H, Zhang C, Wang BJ, Tan XH, Zhang WP, Teng Y, Yang X. Synergistic function of Kras mutation and HBX in initiation and progression of hepatocellular carcinoma in mice. Oncogene. 2014; 33:5133-8.

8. Bernstein E, Duncan EM, Masui O, Gil J, Heard E, Allis CD. Mouse polycomb proteins bind differentially to methylated histone $\mathrm{H} 3$ and RNA and are enriched in facultative heterochromatin. Mol Cell Biol. 2006; 26:2560-9.

9. Kaustov L, Ouyang H, Amaya M, Lemak A, Nady N, Duan S, Wasney GA, Li Z, Vedadi M, Schapira M, Min J, Arrowsmith $\mathrm{CH}$. Recognition and specificity determinants of the human cbx chromodomains. J Biol Chem. 2011; 286:521-9.

10. Senthilkumar R, Mishra RK. Novel motifs distinguish multiple homologues of Polycomb in vertebrates: expansion and diversification of the epigenetic toolkit. BMC Genomics. 2009; 10:549.

11. Klauke K, Radulovic V, Broekhuis M, Weersing E, Zwart E, Olthof S, Ritsema M, Bruggeman S, Wu X, Helin K, Bystrykh L, de Haan G. Polycomb Cbx family members mediate the balance between haematopoietic stem cell selfrenewal and differentiation. Nat Cell Biol. 2013; 15:353-62.

12. Morey L, Helin K. Polycomb group protein-mediated repression of transcription. Trends Biochem Sci. 2010; 35:323-32.

13. Morey L, Pascual G, Cozzuto L, Roma G, Wutz A, Benitah SA, Di Croce L. Nonoverlapping functions of the Polycomb group Cbx family of proteins in embryonic stem cells. Cell Stem Cell. 2012; 10:47-62.

14. Ren X, Kerppola TK. REST interacts with Cbx proteins and regulates polycomb repressive complex 1 occupancy at RE1 elements. Mol Cell Biol. 2011; 31:2100-10.

15. Ren X, Vincenz C, Kerppola TK. Changes in the distributions and dynamics of polycomb repressive complexes during embryonic stem cell differentiation. Mol Cell Biol. 2008; 28:2884-95.

16. Zhen CY, Tatavosian R, Huynh TN, Duc HN, Das R, Kokotovic M, Grimm JB, Lavis LD, Lee J, Mejia FJ, Li Y, Yao T, Ren X. Live-cell single-molecule tracking reveals co-recognition of H3K27me3 and DNA targets polycomb Cbx7-PRC1 to chromatin. 2016; 5.

17. Milosevich N, Gignac MC, McFarlane J, Simhadri C, Horvath S, Daze KD, Croft CS, Dheri A, Quon TT, Douglas SF, Wulff JE, Paci I, Hof F. Selective Inhibition of CBX6: A Methyllysine Reader Protein in the Polycomb Family. ACS Med Chem Lett. 2016; 7:139-44.

18. Richly H, Aloia L, Di Croce L. Roles of the Polycomb group proteins in stem cells and cancer. Cell Death Dis. 2011; 2:e204. 
19. Salama I, Malone PS, Mihaimeed F, Jones JL. A review of the S100 proteins in cancer. Eur J Surg Oncol. 2008; 34:357-64.

20. Cheng S, Zhang X, Huang N, Qiu Q, Jin Y, Jiang D. Downregulation of S100A9 inhibits osteosarcoma cell growth through inactivating MAPK and NF-kappaB signaling pathways. BMC Cancer. 2016; 16:253.

21. Aravalli RN, Cressman EN, Steer CJ. Cellular and molecular mechanisms of hepatocellular carcinoma: an update. Arch Toxicol. 2013; 87:227-47.

22. Giordano S, Columbano A. MicroRNAs: new tools for diagnosis, prognosis, and therapy in hepatocellular carcinoma? Hepatology. 2013; 57:840-7.

23. Aravalli RN, Steer CJ, Cressman EN. Molecular mechanisms of hepatocellular carcinoma. Hepatology. 2008; 48:2047-63.

24. Arzumanyan A, Reis HM, Feitelson MA. Pathogenic mechanisms in HBV- and HCV-associated hepatocellular carcinoma. Nat Rev Cancer. 2013; 13:123-35.

25. Bruix J, Gores GJ, Mazzaferro V. Hepatocellular carcinoma: clinical frontiers and perspectives. Gut. 2014; 63:844-55.

26. Ha NB, Ha NB, Ahmed A, Ayoub W, Daugherty TJ, Chang ET, Lutchman GA, Garcia G, Cooper AD, Keeffe EB, Nguyen MH. Risk factors for hepatocellular carcinoma in patients with chronic liver disease: a casecontrol study. Cancer Causes Control. 2012; 23:455-62.

27. Imamura $H$, Matsuyama $Y$, Tanaka E, Ohkubo $T$, Hasegawa K, Miyagawa S, Sugawara Y, Minagawa M, Takayama T, Kawasaki S, Makuuchi M. Risk factors contributing to early and late phase intrahepatic recurrence of hepatocellular carcinoma after hepatectomy. J Hepatol. 2003; 38:200-7.

28. Li D, Mallory T, Satomura S. AFP-L3: a new generation of tumor marker for hepatocellular carcinoma. Clin Chim Acta. 2001; 313:15-9.

29. Shiraki K, Takase K, Tameda Y, Hamada M, Kosaka Y, Nakano T. A clinical study of lectin-reactive alphafetoprotein as an early indicator of hepatocellular carcinoma in the follow-up of cirrhotic patients. Hepatology. 1995; 22:802-7.

30. Chang KA, Kim HJ, Suh YH. The role of S100a9 in the pathogenesis of Alzheimer's disease: the therapeutic effects of S100a9 knockdown or knockout. Neurodegener Dis. 2012; 10:27-9.

31. Li C, Chen H, Ding F, Zhang Y, Luo A, Wang M, Liu Z. A novel p53 target gene, S100A9, induces p53-dependent cellular apoptosis and mediates the p53 apoptosis pathway. Biochem J. 2009; 422:363-72.
32. Li C, Zhang F, Lin M, Liu J. Induction of S100A9 gene expression by cytokine oncostatin $\mathrm{M}$ in breast cancer cells through the STAT3 signaling cascade. Breast Cancer Res Treat. 2004; 87:123-34.

33. Nacken W, Lekstrom-Himes JA, Sorg C, Manitz MP. Molecular analysis of the mouse S100A9 gene and evidence that the myeloid specific transcription factor C/EBPepsilon is not required for the regulation of the S100A9/A8 gene expression in neutrophils. J Cell Biochem. 2001; 80:606-16.

34. Arai K, Teratani T, Kuruto-Niwa R, Yamada T, Nozawa R. S100A9 expression in invasive ductal carcinoma of the breast: S100A9 expression in adenocarcinoma is closely associated with poor tumour differentiation. Eur J Cancer. 2004; 40:1179-87.

35. Grote J, Konig S, Ackermann D, Sopalla C, Benedyk M, Los M, Kerkhoff C. Identification of poly(ADP-ribose) polymerase- 1 and $\mathrm{Ku} 70 / \mathrm{Ku} 80$ as transcriptional regulators of S100A9 gene expression. BMC Mol Biol. 2006; 7:48.

36. Kerkhoff C, Hofmann HA, Vormoor J, Melkonyan H, Roth J, Sorg C, Klempt M. Binding of two nuclear complexes to a novel regulatory element within the human S100A9 promoter drives the S100A9 gene expression. J Biol Chem. 2002; 277:41879-87.

37. Simard JC, Girard D, Tessier PA. Induction of neutrophil degranulation by S100A9 via a MAPK-dependent mechanism. J Leukoc Biol. 2010; 87:905-14.

38. Fang JY, Richardson BC. The MAPK signalling pathways and colorectal cancer. Lancet Oncol. 2005; 6:322-7.

39. Ghavami S, Chitayat S, Hashemi M, Eshraghi M, Chazin WJ, Halayko AJ, Kerkhoff C. S100A8/A9: a Janusfaced molecule in cancer therapy and tumorgenesis. Eur J Pharmacol. 2009; 625:73-83.

40. Kraunz KS, Nelson HH, Liu M, Wiencke JK, Kelsey KT. Interaction between the bone morphogenetic proteins and Ras/MAP-kinase signalling pathways in lung cancer. Br J Cancer. 2005; 93:949-52.

41. Platanias LC. Map kinase signaling pathways and hematologic malignancies. Blood. 2003; 101:4667-79.

42. Shimo T, Matsumura S, Ibaragi S, Isowa S, Kishimoto K, Mese H, Nishiyama A, Sasaki A. Specific inhibitor of MEK-mediated cross-talk between ERK and p38 MAPK during differentiation of human osteosarcoma cells. J Cell Commun Signal. 2007; 1:103-11. 